\title{
DETERMINATION OF THE HEIGHT OF COUNTERCURRENT SPRAYING EXTRACTORS FOR PURIFICATION OF SEWAGES
}

\author{
A.A.Hasanov, U.R.Guluzade, L.B.Farzaliyeva \\ Azerbaijan State Oil and Industry University \\ alakbar48-48@mail.ru
}

Received 06.03.2018

\begin{abstract}
The mathematical model of countercurrent extractor have been presented with help of which at the first time the average concentrations of components and height of apparatus, being one of the main parameters of extraction process have been determined.
\end{abstract}

Keywords: coefficient, consumption, sewage, extraction, extragent.

\section{Introduction}

Forecasting of the optimal extractors characteristics is actual problem. In connection with this the role of mathematical modeling as the main prerequisite of increase of changes tempos from laboratory plants to apparatuses of large unit power extremely increase.

By satisfactory coincidence of data of modeling and experiment the prerequisites for calculation of optimal versions of extractive apparatuses use are created. The aim of given work - is determination of average concentrations of pollutants, such as butylglycol, phenylisopropanol and acetic acid with help of equations mathematical modeling, of countercurrent extractors being one of the main parameters of extraction process.

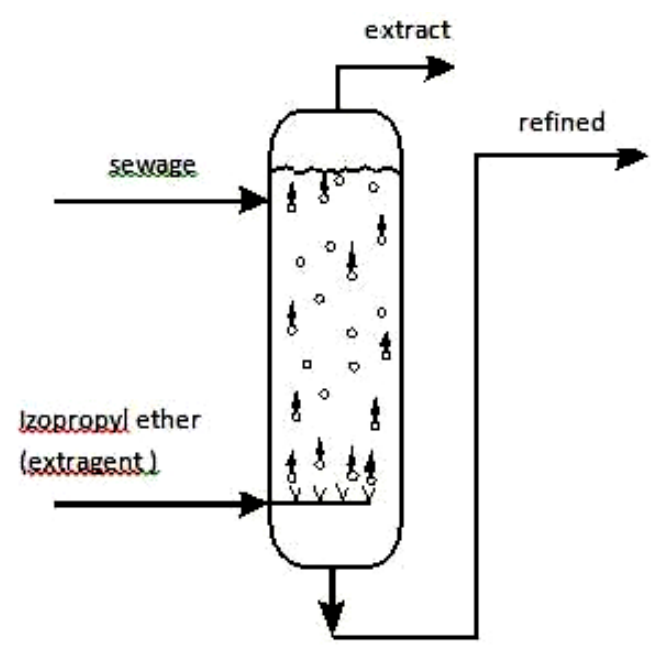

\section{Methods of experiment}

From existing mathematical models for extraction the diffusion and cell models are often used. The first model is used in that case when the object present itself the system with distributed parameters. For cell model is characteristic presentation of object in form of successively connected between oneself the areas with concentrated parameters (Figure 1). As the initial value for modeling the equations system, reflecting a structure of flows in apparatus have been used.

By research of the process of liquid phase extraction by isopropyl ether the samples of sewage containing butylglycol, phenylisopropanol and acetic acid have been used, composition of which is presented in the Table 1. 
Table 1. Composition of waste water

\begin{tabular}{|l|c|c|c|c|c|}
\hline \multirow{2}{*}{$\begin{array}{c}\text { Composition of } \\
\text { waste water }\end{array}$} & \multicolumn{2}{|c|}{ Consumption } & \multicolumn{3}{c|}{ Concentration } \\
\cline { 2 - 6 } & $\mathrm{g} / \mathrm{hour}$ & mol/hour & mass share, weight \% & $\mathrm{g} / \mathrm{dm}^{3}$ & 10.2 \\
\hline butylglycol & 30.6 & 0.339 & 1.02 & 6.5 & 0.113 \\
\hline phenylisopropanol & 19.5 & 0.143 & 0.65 & 8.7 & 0.048 \\
\hline acetic acid & 26.1 & 0.435 & 0.87 & 974.6 & 54.144 \\
\hline water & 2923.8 & 162.433 & 97.46 & 1000.0 & 54.450 \\
\hline at all & 3000.0 & 163.350 & 100.00 & $\mathrm{~mm}^{3}$ \\
\hline
\end{tabular}

\section{Choice of extragent}

Proceeding from solubility of components, selectivity, efficiency, economic profit, coefficient of spreading, regeneration, inter-phase balance, stability, viscosity and toxicity as an extragent the isopropyl ether have been chosen [1,2].

Further the optimal consumption and volume correlation of sewage to isopropyl ether have been calculated. By help of made working like and balance like the amount of stages was found. For that the stages between indicated lines have been constructed and its amount have been determinate, which was equal 3 .

\section{Choice of extractor}

By choice of extractor by us a large number of requirements, presented to apparatuses $[3,4]$ such as productivity, direction of masstransfer, correlation of flows, lightness of operation, containment ability, physical properties of liquids, number of theoretical stages, tendency to emulsification etc. have been taken into consideration. On base of received results it have been determined that the most suitable for our researchs is spraying extractive columns.

By extraction of multicomponent systems it is necessary to take into consideration a possibility change of rates feed of solid, dispersed phases and coefficient of mass-transfer by height of column. For given case the change of balanced concentrations by height of column is determined not only by correlation of feed rates of over-all (water) and dispersed (isopropyl ether) phases, but also by values of mass-transfer for every from extracted components. Therefore, in total case, the rate of mass-transfer for the all column it is not to present in form of product of mass-transfer coefficient on average moving force [5].

On the basis of received data the ma- thematical model of extraction process for determination of average concentrations of components in cells have been developed [6]. It is a system of differential equations of material balance, determing the interphase spraying of pollutant components concentrations in cells by height of column [2].

The kinetic method calculation have been developed in supposition, that the flow rate and coefficient of mass-transfer don't are changed by column height (Figure 2).

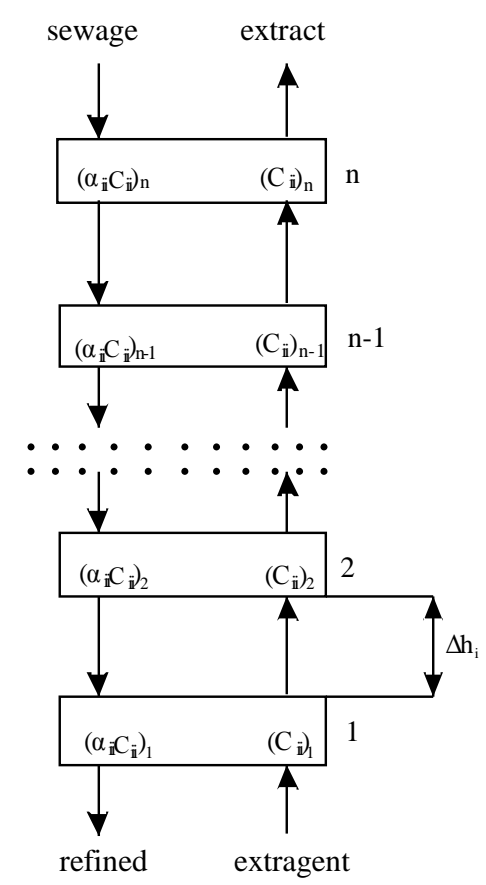

Fig. 2. Structure of flows in apparatus.

In given work the method calculation of average concentration of components by height of column by use of the following equations of mathematic modeling is stated: 


$$
\begin{aligned}
& \left(C_{1}\right)_{i}^{\mathrm{av}}=\frac{1}{h_{i}-h_{i-1}} \int_{h_{i-1}}^{h_{i}}\left(C_{1}\right)_{i} h \cdot \mathrm{d} h=\left(\alpha_{1} C_{1}\right)_{i}-\left[\left(\alpha_{1} C_{1}\right)_{i}-\left(C_{1}\right)_{i-1}\right] \frac{\varepsilon U_{d}^{0}}{K_{1} F \Delta h_{i}}\left(1-\mathrm{e}^{-\frac{K_{1} F}{\varepsilon U_{d}^{0} \Delta h_{1}}}\right), \\
& \left(C_{2}\right)_{i}^{\mathrm{av}}=\frac{1}{h_{i}-h_{i-1}} \int_{h_{i-1}}^{h_{i}}\left(C_{2}\right)_{i} h \cdot \mathrm{d} h=\left(\alpha_{2} C_{2}\right)_{i}-\left[\left(\alpha_{2} C_{2}\right)_{i}-\left(C_{2}\right)_{i-1}\right] \frac{\varepsilon U_{d}^{0}}{K_{2} F \Delta h_{i}}\left(1-\mathrm{e}^{-\frac{K_{1} F}{\varepsilon U_{d}^{0} \Delta h_{1}}}\right), \\
& \left(C_{3}\right)_{i}^{\mathrm{av}}=\frac{1}{h_{i}-h_{i-1}} \int_{h_{i-1}}^{h_{i}}\left(C_{3}\right)_{i} h \cdot \mathrm{d} h=\left(\alpha_{3} C_{3}\right)_{i}-\left[\left(\alpha_{3} C_{3}\right)_{i}-\left(C_{3}\right)_{i-1}\right] \frac{\varepsilon U_{d}^{0}}{K_{3} F \Delta h_{i}}\left(1-\mathrm{e}^{-\frac{K_{3} F}{\varepsilon U_{d}^{0} \Delta h_{1}}}\right) .
\end{aligned}
$$

By integration of these equations:

$$
\begin{aligned}
& \left(C_{1}\right)_{i}^{\mathrm{av}}=\left(\alpha_{1} C_{1}\right)_{i}-\left[\left(\alpha_{1} C_{1}\right)_{i}-\left(C_{1}\right)_{i-1}\right] \frac{\varepsilon U_{d}^{0}}{K_{1} F \Delta h_{1}}\left(1-\mathrm{e}^{-\frac{K_{1} F}{\varepsilon U_{d}^{0} \Delta h_{1}}}\right), \\
& \left(C_{2}\right)_{i}^{\mathrm{av}}=\left(\alpha_{2} C_{2}\right)_{i}-\left[\left(\alpha_{2} C_{2}\right)_{i}-\left(C_{2}\right)_{i-1}\right] \frac{\varepsilon U_{d}^{0}}{K_{2} F \Delta h_{i}}\left(1-\mathrm{e}^{-\frac{K_{2} F}{\varepsilon U_{d}^{0} \Delta h_{1}}}\right), \\
& \left(C_{3}\right)_{i}^{\mathrm{av}}=\left(\alpha_{3} C_{3}\right)_{i}-\left[\left(\alpha_{3} C_{3}\right)_{i}-\left(C_{3}\right)_{i-1}\right] \frac{\varepsilon U_{d}^{0}}{K_{3} F \Delta h_{i}}\left(1-\mathrm{e}^{-\frac{K_{3} F}{\varepsilon U_{d}^{0} \Delta h_{1}}}\right),
\end{aligned}
$$

where $C_{1}, C_{2}, C_{3}$ - are the concentrations, of butylglycol, phenylisopropanol and acetic acid in cells correspondingly; $\left(C_{1}\right)_{i}^{\mathrm{av}}, \quad\left(C_{2}\right)_{i}^{\mathrm{av}}$ and $\left(C_{3}\right)_{i}^{\text {av }}$ - are the average concentrations of the same components in cells; $i$ - number of cells; $\alpha_{1}, \alpha_{2}, \alpha_{3}-$ are the coefficients spraying of components between phases; $\varepsilon-$ share of disperse phase; $h$ - height of cells; $U_{d}^{0}$ - rate of disperse phase; $F$ - square of specific contact surface; $F=\frac{6 \varepsilon}{d_{k}}$, where $d_{k}$ - diameter of drop; $\Delta h_{i}$ - distance between cells which is determined by V.V.Kafarov formula [7]. The numerical values of high mentioned parameters are shown in the work [6]. Calculation of coefficients mass-transfer of $i$-component $\left(K_{i}\right)$ are shown in the works [8,9]. $K_{1}, K_{2}, K_{3}$ coefficients of mass-transfer of butylglycol, phenylisopropanol and acetic acid correspondingly and are determined by equation [9].

$$
K_{i}=\left(\frac{1}{\beta_{i, C}}+\frac{\alpha_{i}}{\beta_{i, D}}\right)^{-1},
$$

where $\alpha_{i}-$ constant of phase balance of components $\alpha_{1}=0.2140, \alpha_{2}=0.2142, \alpha_{3}=0.2136$; $\beta_{i, C}$ and $\beta_{i, D}-$ coefficients of mass-output of components in overall and dispersed phases correspondingly.
These coefficients are determined by formulas

$$
\begin{aligned}
\beta_{i, D} & =\frac{2 p^{2} \cdot D_{i, D}}{3 d_{k}}, \\
\beta_{i, C} & =\frac{2 p^{2} \cdot D_{i, C}}{3 d_{k}},
\end{aligned}
$$

where - $D_{i, C}, D_{i, D}$ are the coefficients of molecular diffusion of components in dispersed and overall phases correspondingly. These coefficients are determined by formulas

$$
\begin{aligned}
& D_{i, D}=\frac{7.4 \cdot 10^{-8}\left(\varphi_{D} \cdot M_{D}\right)^{0.5} \cdot T}{M_{D} \cdot V_{i}^{0.6}}, \\
& D_{i, C}=\frac{7.4 \cdot 10^{-8}\left(\varphi_{C} M_{C}\right)^{0.5} \cdot T}{M_{C} \cdot V_{i}^{0.6}},
\end{aligned}
$$

where $-\varphi_{D}$ and $\varphi_{C}-$ are factors of associations in dispers and overall phases, $\varphi_{D}=1.0, \varphi_{C}=2.6$ [10], $T$ - absolute temperature, $T=293 \mathrm{~K} ; M_{D}$ and $M_{C}$ - molecular mass of disperse and overall phases $M_{D}=M_{\mathrm{C}_{4} \mathrm{H}_{10} \mathrm{O}_{2}}=102 ; M_{C}=M_{\mathrm{H}_{2} \mathrm{O}}=18$; $V_{i}$ - mole volume of $i$-component, $\mathrm{cm}^{3} /$ mole; $M_{D}$ and $M_{C}-$ dynamic viscosity of disperse and overall phases; $M_{D}=0.31 s P ; M_{C}=1 s P$. The calculation values of high mentioned parameters are shown in the Table 2. 
Proceeding from high calculated values of parameters the following are received for

butylglycol

$$
\begin{aligned}
& e^{-\frac{4.28 \cdot 10^{-5} \cdot 0.05}{0.002 \cdot 0.0044}}=0.98, \\
& e^{-\frac{3.09 \cdot 10^{-5} \cdot 0.05}{0.02 \cdot 0.0044}}=0.98, \\
& e^{-\frac{6.08 \cdot 10^{-5}}{0.02 \cdot 0.0044}}=0.97 .
\end{aligned}
$$$$
\text { phenylisopropanol } \quad e^{-\overline{0.02 \cdot 0.0044}}=0.98 \text {, }
$$$$
\text { acetic acid }
$$

It is necessary to notice that by calculation of average concentrations in cells by formula (1)-(3) the experimental values of components concentrations have been used, which are shown in the Table 3.
As it is evident from data, presented in the tables, extraction degrees in the first extractive column make up: by butylglycol $-70.92 \%$, by phenylisopropanol $-96.24 \%$ by acetic acid $66.52 \%$ [11]. From these data it is possible to conclude, that it is necessary to continue process in the second extractive column (Table 4).

The data received after second extractor determined on necessity continuation of process on the third stage, where the complete purification of sewage is achieved (Table 5).

Table 6.

Indices by stages are presented in the

Table 2. Coefficients of molecular diffusion, massoutput, mass-transfer and spraying of component

\begin{tabular}{|c|c|c|c|c|c|c|c|c|c|}
\hline \multirow{4}{*}{$\begin{array}{l}\text { № } \\
\text { cells }\end{array}$} & \multicolumn{3}{|c|}{ Butylglycol } & \multicolumn{3}{|c|}{ Phenylisopropanol } & \multicolumn{3}{|c|}{ Acetic acid } \\
\hline & \multicolumn{9}{|c|}{$\mathrm{mol} / \mathrm{dm}^{3}$} \\
\hline & \multicolumn{2}{|c|}{$\begin{array}{l}\text { experimental values in } \\
\text { phases }\end{array}$} & \multirow{2}{*}{$\begin{array}{l}\text { average } \\
\text { calcu- } \\
\text { lation } \\
\end{array}$} & \multicolumn{2}{|c|}{$\begin{array}{c}\text { experimental values in } \\
\text { phases }\end{array}$} & \multirow{2}{*}{$\begin{array}{l}\text { average } \\
\text { calcu- } \\
\text { lation } \\
\end{array}$} & \multicolumn{2}{|c|}{$\begin{array}{l}\text { experimental values } \\
\text { in phases }\end{array}$} & \multirow{2}{*}{$\begin{array}{l}\text { average } \\
\text { calcu- } \\
\text { lation }\end{array}$} \\
\hline & $\begin{array}{c}\text { in } \\
\text { extract }\end{array}$ & $\begin{array}{l}\text { in refined } \\
\text { oil }\end{array}$ & & $\begin{array}{c}\text { in } \\
\text { extract }\end{array}$ & $\begin{array}{l}\text { in refined } \\
\text { oil }\end{array}$ & & $\begin{array}{c}\text { in } \\
\text { extract }\end{array}$ & $\begin{array}{l}\text { in refined } \\
\text { oil }\end{array}$ & \\
\hline 1 & 0.881 & 0.033 & 0.0058 & 0.377 & 0.014 & 0.0003 & 0.236 & 0.051 & 0.0066 \\
\hline 2 & 0.933 & 0.035 & 0.7465 & 0.399 & 0.015 & 0.3697 & 1.305 & 0.053 & 1.0827 \\
\hline 3 & 0.988 & 0.038 & 0.7909 & 0.423 & 0.016 & 0.3918 & 1.376 & 0.056 & 1.1423 \\
\hline 4 & 1.045 & 0.040 & 0.8312 & 0.449 & 0.017 & 0.4154 & 1.452 & 0.059 & 1.2052 \\
\hline 5 & 1.103 & 0.042 & 0.8880 & 0.476 & 0.018 & 0.4403 & 1.532 & 0.063 & 1.2716 \\
\hline 6 & 1.169 & 0.044 & 0.9409 & 0.504 & 0.019 & 0.4667 & 1.617 & 0.066 & 1.3417 \\
\hline 7 & 1.236 & 0.047 & 0.9971 & 0.536 & 0.020 & 0.4946 & 1.706 & 0.070 & 1.4156 \\
\hline 8 & 1.310 & 0.050 & 1.0565 & 0.567 & 0.022 & 0.5243 & 1.800 & 0.074 & 1.4935 \\
\hline 9 & 1.387 & 0.053 & 1.1195 & 0.601 & 0.023 & 0.5558 & 1.899 & 0.078 & 1.5758 \\
\hline 10 & 1.469 & 0.056 & 1.1863 & 0.637 & 0.024 & 0.5892 & 2.003 & 0.082 & 1.6627 \\
\hline 11 & 1.556 & 0.059 & 1.2570 & 0.675 & 0.026 & 0.6243 & 2.114 & 0.087 & 1.7543 \\
\hline 12 & 1.647 & 0.062 & 1.3319 & 0.715 & 0.027 & 0.6619 & 2.230 & 0.091 & 1.8509 \\
\hline 13 & 1.744 & 0.056 & 1.4113 & 0.758 & 0.029 & 0.7017 & 2.353 & 0.096 & 1.9528 \\
\hline 14 & 1.845 & 0.070 & 1.4954 & 0.804 & 0.031 & 0.7337 & 2.483 & 0.102 & 2.0603 \\
\hline 15 & 1.954 & 0.074 & 1.5845 & 0.852 & 0.034 & 0.7883 & 2.620 & 0.107 & 2.1739 \\
\hline 16 & 2.069 & 0.079 & 1.6790 & 0.903 & 0.032 & 0.8357 & 2.764 & 0.113 & 2.2938 \\
\hline 17 & 2.184 & 0.083 & 1.7791 & 0.957 & 0.036 & 0.8859 & 2.916 & 0.119 & 2.4202 \\
\hline 18 & 2.311 & 0.088 & 1.8852 & 1.015 & 0.039 & 0.9390 & 3.077 & 0.126 & 2.5536 \\
\hline 19 & 2.447 & 0.093 & 1.9977 & 1.076 & 0.041 & 0.9954 & 3.247 & 0.133 & 2.6444 \\
\hline 20 & 2.589 & 0.098 & 2.1169 & 1.140 & 0.043 & 1.0552 & 3.426 & 0.140 & 2.8429 \\
\hline 21 & 2.734 & 0.104 & 2.2431 & 1.172 & 0.045 & 1.1186 & 3.614 & 0.148 & 2.9995 \\
\hline 22 & 2.898 & 0.110 & 2.3766 & 1.242 & 0.047 & 1.1495 & 3.614 & 0.48 & 2.9995 \\
\hline
\end{tabular}

\begin{tabular}{|l|c|c|c|c|c|c|c|c|c|}
\hline \multirow{2}{*}{ Component } & $D_{i, C} \cdot 10^{9}$ & $D_{i, D} \cdot 10^{9}$ & $\beta_{i, D} \cdot 10^{5}$ & $\beta_{i, C} \cdot 10^{5}$ & $K_{i, C} \cdot 10^{5}$ & $K_{i, D} \cdot 10^{5}$ & $K_{i} \cdot 10^{5}$ & $\alpha_{i}$ \\
\cline { 2 - 10 } & 0.894 & 4.18 & 0.687 & 0.147 & 0.147 & 0.687 & 0.141 & 0.2139 \\
\hline butylglycol & 0.658 & 3.07 & 0.504 & 0.108 & 0.108 & 0.504 & 0.103 & 0.2142 \\
\hline phenylisopropanol & 0.658 & 5.98 & 0.983 & 0.210 & 0.210 & 0.983 & 0.201 & 0.2136 \\
\hline acetic acid & 1.280 &
\end{tabular}

Table 3. Experimental values of components concentrations 
Table 4. Concentration of components in cells in the second extractor

\begin{tabular}{|c|c|c|c|c|c|c|c|c|c|}
\hline \multirow{4}{*}{$\begin{array}{c}\text { № } \\
\text { cells }\end{array}$} & \multicolumn{3}{|c|}{ Butylglycol } & \multicolumn{3}{|c|}{ Phenylisopropanol } & \multicolumn{3}{|c|}{ Acetic acid } \\
\hline & \multicolumn{9}{|c|}{$\mathrm{mol} / \mathrm{dm}^{3}$} \\
\hline & \multicolumn{2}{|c|}{$\begin{array}{l}\text { experimental values in } \\
\text { phases }\end{array}$} & \multirow{2}{*}{$\begin{array}{l}\text { average } \\
\text { calcu- } \\
\text { lation }\end{array}$} & \multicolumn{2}{|c|}{$\begin{array}{l}\text { experimental values in } \\
\text { phases }\end{array}$} & \multirow{2}{*}{$\begin{array}{l}\text { average } \\
\text { calcu- } \\
\text { lation }\end{array}$} & \multicolumn{2}{|c|}{$\begin{array}{l}\text { experimental values } \\
\text { in phases }\end{array}$} & \multirow{2}{*}{$\begin{array}{l}\text { average } \\
\text { calcu- } \\
\text { lation }\end{array}$} \\
\hline & $\begin{array}{c}\text { in } \\
\text { extract }\end{array}$ & $\begin{array}{c}\text { in refined } \\
\text { oil }\end{array}$ & & $\begin{array}{c}\text { in } \\
\text { extract }\end{array}$ & $\begin{array}{l}\text { in refined } \\
\text { oil }\end{array}$ & & $\begin{array}{c}\text { in } \\
\text { extract }\end{array}$ & $\begin{array}{l}\text { in refined } \\
\text { oil }\end{array}$ & \\
\hline 1 & 0.2889 & 0.00918 & 0.0019 & 0.2889 & 0.0092 & 0.0003 & 0.3810 & 0.0110 & 0.002 \\
\hline 2 & 0.2975 & 0.0112 & 0.2443 & 0.2919 & 0.0089 & 0.2894 & 0.3980 & 0.0112 & 0.3406 \\
\hline 3 & 0.3188 & 0.0118 & 0.2597 & 0.3190 & 0.126 & 0.3096 & 0.4191 & 0.0158 & 0.3577 \\
\hline 4 & 0.3412 & 0.0101 & 0.2750 & 0.3410 & 0.0136 & 0.3290 & 0.4451 & 0.0168 & 0.3758 \\
\hline 5 & 0.3650 & 0.0131 & 0.2918 & 0.3618 & 0.0136 & 0.3290 & 0.4696 & 0.0179 & 0.3948 \\
\hline 6 & 0.3819 & 0.0139 & 0.3097 & 0.3810 & 0.0136 & 0.3290 & 0.4974 & 0.0189 & 0.4146 \\
\hline 7 & 0.4015 & 0.0110 & 0.3286 & 0.4096 & 0.0136 & 0.3290 & 0.5186 & 0.0198 & 0.4355 \\
\hline 8 & 0.4339 & 0.0170 & 0.3486 & 0.4310 & 0.0136 & 0.3290 & 0.5396 & 0.0202 & 0.4575 \\
\hline 9 & 0.4610 & 0.0182 & 0.3699 & 0.4610 & 0.0136 & 0.3290 & 0.5650 & 0.0218 & 0.4806 \\
\hline 10 & 0.4890 & 0.0194 & 0.3925 & 0.4856 & 0.0136 & 0.3290 & 0.5912 & 0.0225 & 0.5046 \\
\hline 11 & 0.5115 & 0.0196 & 0.4165 & 0.5190 & 0.0136 & 0.3290 & 0.6219 & 0.0232 & 0.5304 \\
\hline 12 & 0.5569 & 0.0198 & 0.4419 & 0.5588 & 0.0136 & 0.3290 & 0.6519 & 0.0251 & 0.5572 \\
\hline 13 & 0.5844 & 0.0202 & 0.4690 & 0.5892 & 0.0136 & 0.3290 & 0.6826 & 0.0268 & 0.5852 \\
\hline 14 & 0.6219 & 0.0199 & 0.4977 & 0.6260 & 0.0136 & 0.3290 & 0.7632 & 0.0276 & 0.6152 \\
\hline 15 & 0.6585 & 0.0241 & 0.5281 & 0.6587 & 0.0136 & 0.3290 & 0.7912 & 0.0298 & 0.6460 \\
\hline 16 & 0.6980 & 0.0236 & 0.5603 & 0.7019 & 0.0136 & 0.3290 & 0.8462 & 0.0311 & 0.6787 \\
\hline 17 & 0.7495 & 0.0249 & 0.5946 & 0.7830 & 0.0136 & 0.3290 & 0.8819 & 0.0325 & 0.7130 \\
\hline 18 & 0.7894 & 0.0301 & 0.6301 & 0.7850 & 0.0136 & 0.3290 & 1.0711 & 0.0335 & 0.7491 \\
\hline 19 & 0.8315 & 0.0298 & 0.7102 & 0.8370 & 0.0136 & 0.3290 & 1.1260 & 0.0412 & 0.7877 \\
\hline 20 & 0.8371 & 0.0316 & 0.7102 & 0.8370 & 0.0136 & 0.3290 & 1.0098 & 0.0421 & 0.9474 \\
\hline 21 & 0.8371 & 0.0316 & 0.7102 & 0.8370 & 0.0136 & 0.3290 & 1.2414 & 0.0436 & 0.9960 \\
\hline 22 & 0.8371 & 0.0316 & 0.7102 & 0.8370 & 0.0136 & 0.3290 & 1.2512 & 0.0464 & 1.046 \\
\hline
\end{tabular}

Table 5. Concentration of components in cells in the third extractor

\begin{tabular}{|c|c|c|c|c|c|c|c|c|c|}
\hline \multirow{4}{*}{$\begin{array}{c}\text { № } \\
\text { cells }\end{array}$} & \multicolumn{3}{|c|}{ Butylglycol } & \multicolumn{3}{|c|}{ Phenylisopropanol } & \multicolumn{3}{|c|}{ Acetic acid } \\
\hline & \multicolumn{9}{|c|}{$\mathrm{mol} / \mathrm{dm}^{3}$} \\
\hline & \multicolumn{2}{|c|}{$\begin{array}{c}\text { experimental values } \\
\text { in phases }\end{array}$} & \multirow{2}{*}{$\begin{array}{l}\text { average } \\
\text { calcu- } \\
\text { lation }\end{array}$} & \multicolumn{2}{|c|}{$\begin{array}{l}\text { experimental values in } \\
\text { phases }\end{array}$} & \multirow{2}{*}{$\begin{array}{l}\text { average } \\
\text { calcu- } \\
\text { lation }\end{array}$} & \multicolumn{2}{|c|}{$\begin{array}{c}\text { experimental values } \\
\text { in phases }\end{array}$} & \multirow{2}{*}{$\begin{array}{c}\text { average } \\
\text { calcu- } \\
\text { lation }\end{array}$} \\
\hline & $\begin{array}{c}\text { in } \\
\text { extract }\end{array}$ & $\begin{array}{l}\text { in refined } \\
\text { oil }\end{array}$ & & $\begin{array}{c}\text { in } \\
\text { extract }\end{array}$ & $\begin{array}{l}\text { in refined } \\
\text { oil }\end{array}$ & & $\begin{array}{c}\text { in } \\
\text { extract }\end{array}$ & $\begin{array}{l}\text { in refined } \\
\text { oil }\end{array}$ & \\
\hline 1 & 0.0381 & 0.00051 & $0.6 \cdot 10^{-4}$ & 0.0110 & 0.00011 & $4.5 \cdot 10^{-4}$ & 0.0498 & 0.0006 & $0.9 \cdot 10^{-4}$ \\
\hline 2 & 0.0379 & 0.00062 & 0.0 .381 & 0.0198 & 0.00029 & 0.0084 & 0.0641 & 0.00088 & 0.0439 \\
\hline 3 & 0.0415 & 0.00069 & 0.0473 & 0.0368 & 0.00056 & 0.0175 & 0.0699 & 0.00094 & 0.0580 \\
\hline 4 & 0.0568 & 0.00091 & 0.0526 & 0.0694 & 0.00098 & 0.0346 & 0.0798 & 0.00098 & 0.0644 \\
\hline 5 & 0.0649 & 0.00092 & 0.0650 & 0.0892 & 0.00112 & 0.0547 & 0.0886 & 0.00110 & 0.0717 \\
\hline 6 & 0.0788 & 0.00100 & 0.0723 & 0.0996 & 0.00126 & 0.0677 & 0.00994 & 0.00112 & 0.0797 \\
\hline 7 & 0.0912 & 0.00118 & 0.0804 & 0.1256 & 0.00169 & 0.0795 & 0.1022 & 0.00121 & 0.0886 \\
\hline 8 & 0.1086 & 0.00121 & 0.0893 & 0.1498 & 0.00188 & 0.0963 & 0.1168 & 0.00142 & 0.0984 \\
\hline 9 & 0.1328 & 0.00180 & 0.1103 & 0.1784 & 0.00189 & 0.1156 & 0.1268 & 0.00176 & 0.1094 \\
\hline 10 & 0.1418 & 0.00189 & 0.1363 & 0.1894 & 0.00286 & 0.1326 & 0.1478 & 0.00194 & 0.1216 \\
\hline 11 & 0.1699 & 0.00212 & 0.1514 & 0.2101 & 0.000298 & 0.1437 & 0.1689 & 0.00215 & 0.1352 \\
\hline 12 & 0.1812 & 0.00244 & 0.1683 & 0.2409 & 0.00262 & 0.1616 & 0.1876 & 0.00226 & 0.1502 \\
\hline 13 & 0.2216 & 0.00298 & 0.1871 & 0.2910 & 0.00312 & 0.1801 & 0.2021 & 0.00316 & 0.1670 \\
\hline 14 & 0.2822 & 0.00365 & 0.2311 & 0.3168 & 0.00388 & 0.1977 & 0.2284 & 0.00332 & 0.1856 \\
\hline 15 & 0.3465 & 0.00456 & 0.2855 & 0.3402 & 0.00415 & 0.2172 & 0.2586 & 0.00398 & 0.2062 \\
\hline 16 & 0.3912 & 0.00499 & 0.3526 & 0.3650 & 0.00426 & 0.2342 & 0.2812 & 0.00465 & 0.2292 \\
\hline 17 & 0.4410 & 0.00568 & 0.3918 & 0.3650 & 0.00489 & 0.2516 & 0.3519 & 0.00579 & 0.2548 \\
\hline 18 & 0.4956 & 0.00686 & 0.4354 & 0.3650 & 0.00489 & 0.2516 & 0.4378 & 0.00628 & 0.3147 \\
\hline 19 & 0.5486 & 0.00716 & 0.4838 & 0.3650 & 0.00489 & 0.2516 & 0.5382 & 0.00992 & 0.3885 \\
\hline 20 & 0.6118 & 0.00789 & 0.5376 & 0.3650 & 0.00489 & 0.2516 & 0.7715 & 0.01128 & 0.4798 \\
\hline 21 & 0.6492 & 0.00868 & 0.5973 & 0.3650 & 0.00489 & 0.2516 & 0.8522 & 0.01368 & 0.6816 \\
\hline 22 & 0.7124 & 0.00992 & 0.6289 & 0.3650 & 0.00489 & 0.2516 & 1.0208 & 0.01401 & 0.7576 \\
\hline
\end{tabular}


Table 6. Indices of liquid phase extraction by stages

\begin{tabular}{|c|c|c|c|c|}
\hline \multirow{2}{*}{ 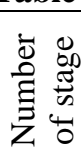 } & \multirow{2}{*}{$\begin{array}{c}\text { Consumption } \\
\text { of extragent } \\
\mathrm{cm}^{3} / \text { hour }\end{array}$} & \multicolumn{3}{|c|}{ Extraction degree, mas $\%$} \\
\hline & & $\begin{array}{l}\text { butyl- } \\
\text { glycol }\end{array}$ & $\begin{array}{c}\text { phenyliso- } \\
\text { propanol }\end{array}$ & $\begin{array}{c}\text { acetic } \\
\text { acid }\end{array}$ \\
\hline 1 & 80 & 70.92 & 69.24 & 66.52 \\
\hline 2 & 80 & 20.00 & 19.94 & 23.10 \\
\hline 3 & 40 & 8.59 & 10.48 & 9.87 \\
\hline
\end{tabular}

As it is evident in the all three extractors there are 22 cells. Owing to distance between cells $\Delta h_{i}=0.05 \mathrm{~m}$, then height of apparatuses make up: $H_{1}=H_{2}=H_{3}=22 \cdot \Delta h_{i}=22 \cdot 0.05=1.1 \mathrm{~m}$

\section{Conclusion}

The extraction of butylglycol, phenylisopropanol and acetic acid from water solution in spraying column have been researched.

The concentration of components in cells by height of column and extraction degree in the all extractors are experimentally determined.

The number of cells in extractor have been found.

The mathematical model have been proposal by help of which at the first time the average concentration of components have been determined.

On base of average concentration of components in cells the high of extractor have been determined.

It have been proved that equations of mass-transfer for every component may be decided independently from each another, by that decision of the task of multicomponent extraction come to decision of the task of one component extraction.

\section{References}

1. Gasanov A.A. Mathematical modeling of ex- traction processes in spray extraction column // European applied sciences. 2014. No 4. P. 96-100.

2. Kelbaliev G.I., Suleimanov G.Z., Fariborz A. Zoroufi, Gasanov A.A. Modelirovanie protcessov zhidkofaznoi ekstraktcionnoi ochistki stochnykh vod // V Mire Nauchnykh otkrytii. Nauchnoinnovatcionnyi centr, Krasnoiarsk. 2011. Vyp. 2. S. $168-170$.

3. Zhidkostnaia ekstraktciia (teoriia i praktika) / Pod red. Kasatkina A.G. M.: Gos. nauch.-tekhn. izd-vo him. lit-ry. 1958. $155 \mathrm{~s}$.

4. Ziulkovskii Z. Zhidkostnaia ekstraktciia v himicheskoi promyshlennosti. L.: Goshimzdat, 1963. $480 \mathrm{~s}$.

5. Pat. 201001176039 USA. Treatment method of organic compounds included in waste water, a treatment apparatus of organic compounds included in waste water, a treatment system of organic compounds included in waste water and a bitumen collecting system / Honji A., Ishii H., Mochizuki A., Saho N. 2010.

6. Gasanov A.A. Liquid Extraction Treatment of Wastewaters at Paint and Varnish Production Facilities Using the Spray Column // Water Chem. and Technol. 2016. V. 38. No 4. P. 407-419.

7. Kafarov V.V., Perov V.L., Meshalkin V.P. Printcipy matematicheskogo modelirovaniia himikotekhnologicheskikh sistem. M.: Himiia, 1974. 344 s.

8. Gasanov A.A., Suleimanov G.Z., Kelbaliev G.I. Liquid-Phase Extraction Treatment of Wastewater of Paint and Varnish Production Facilities in the Extractor with Mixer // Water Chem. and Technol. 2016. V. 38. No 6. P. 336-341.

9. Dytnerskii Iu.I. Protcessy i apparaty himicheskoi tekhnologii. M.: Himiia, 1995. 768 s.

10. Brounshtein B.I., Shchegolev V.V. Gidrodinamika, masso- i teploobmen v kolonnykh apparatakh. L.: Himiia, 1988. 277 s.

11. Gasanov A.A. Matematicheskoe opisanie protcessa zhidkofaznoi ekstraktcii stochnykh vod // VIII Mezhdunarodnyi simpozium "Fundamentalnye i pricladnye problemy nauki". Moskva. 2013. Materialy simpoziuma. T. 4. S. 52-60.

\section{TULLANTI SULARINI TӘMIZLӘMӘK ÜÇÜN ӘKS AXINLI SəPӘLӘYİCİ EKSTRAKTORUN HÜNDÜRLÜYÜNÜN TOYINII \\ O.A.Həsənov, Ü.R.Quluzadə, L.B.Fərzəliyeva}

Differensial tənliklər əsasında əks axınlı ekstraksiya aparatının hündürlüyünü təyin etməyə imkan verən riyazi model verilmişdir.

Açar sözlor: amsal, sarf, tullantı suyu, ekstraksiya, ekstragent.

ОПРЕДЕЛЕНИЕ ВЫСОТЫ ПРОТИВОТОЧНОГО РАСПЫЛИТЕЛЬНОГО ЭКСТРАКТОРА ДЛЯ ОЧИСТКИ СТОЧНЫХ ВОД

\section{А.А.Гасанов, У.Р.Гулузаде, Л.Б.Фарзалиева}

Представлена математическая модель противоточного экстрактора, основанная на дифференциальных уравнениях, позволяющая определять средние концентрации компонентов и высоту аппарата.

Ключевые слова: коэффициент, расход, сточная вода, экстракция, экстрагент. 